\title{
Sensitive Method for Quantification of Octamethylcyclotetrasiloxane (D4) and Decamethylcyclopentasiloxane (D5) in End-Exhaled Air by Thermal Desorption Gas Chromatography Mass Spectrometry
}

\author{
Jacqueline W. H. Biesterbos, ${ }^{* \dagger}{ }^{\dagger}$ Gwendolyn Beckmann, ${ }^{\dagger}$ Rob B. M. Anzion, ${ }^{\dagger}$ Ad M. J. Ragas, ${ }^{\dagger}$ \\ Frans G. M. Russel, ${ }^{\S}$ and Paul T. J. Scheepers ${ }^{\dagger}$ \\ ${ }^{\dagger}$ Department for Health Evidence and ${ }^{\S}$ Department of Pharmacology and Toxicology, Radboud University Medical Center, \\ P.O. Box 9101, 6500 HB Nijmegen, The Netherlands \\ ${ }^{\ddagger}$ Department of Environmental Science, Institute for Wetland and Water Research, Radboud University Nijmegen, \\ P.O. Box 9010, 6500 GL Nijmegen, The Netherlands
}

ABSTRACT: Octamethylcyclotetrasiloxane (D4) and decamethylpentasiloxane (D5) are used as ingredients for personal care products (PCPs). Because of the use of these PCPs, consumers are exposed daily to D4 and D5. A sensitive analytical method was developed for analysis of D4 and D5 in end-exhaled air by thermal desorption gas chromatography mass spectrometry (TD-GC-MS), to determine the internal dose for consumer exposure assessment. Fifteen consumers provided end-exhaled air samples that were collected using Bio-VOC breath samplers and subsequently transferred to automatic thermal desorption (ATD) tubes. Prior to use, the ATD tubes were conditioned for a minimum of $4 \mathrm{~h}$ at $350{ }^{\circ} \mathrm{C}$.

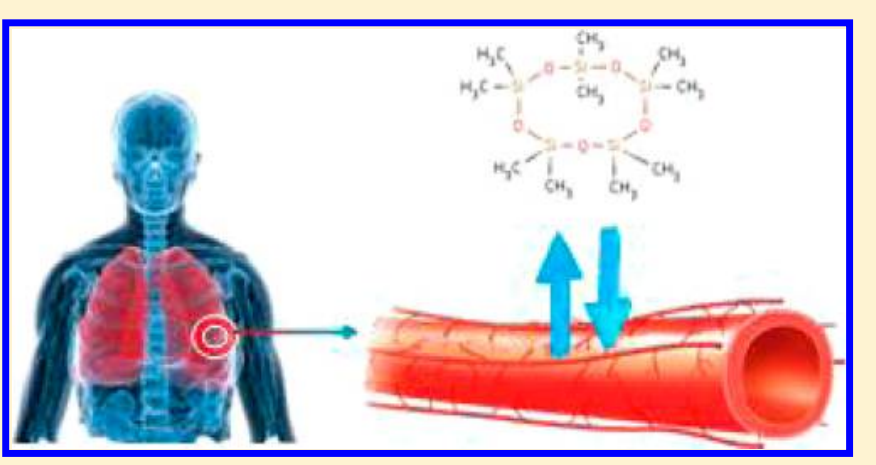
The TD unit and auto sampler were coupled to a GC-MS using electron ionization. Calibration was performed using $0-10 \mathrm{ng} / \mu \mathrm{L}$ solutions of D4/D5 and ${ }^{13} \mathrm{C}$-labeled D4/D5 as internal standards. The ions monitored were $\mathrm{m} / z 281 \mathrm{for} \mathrm{D} 4$, 355 for D5, 285 for ${ }^{13} \mathrm{C}$-labeled D4, and 360 for ${ }^{13} \mathrm{C}$-labeled D5. The addition of internal standard reduced the coefficient of variation from $30.8 \%$ to $9.5 \%$ for D4 and from $37.8 \%$ to $12.5 \%$ for D5. The limit of quantification was $2.1 \mathrm{ng} / \mathrm{L}$ end-exhaled air for D4 and $1.4 \mathrm{ng} / \mathrm{L}$ end-exhaled air for D5. With this method, cyclic siloxanes (D4 and D5) can be quantified in end-exhaled air at concentrations as low as background levels observed in the general population.

$\mathrm{O}$ $\mathrm{n}$ a daily basis, consumers are exposed to cyclic siloxanes, such as octamethylcyclotetrasiloxane (D4) and decamethylcyclopentasiloxane (D5), via the use of personal care products (PCPs). ${ }^{1,2}$ These substances are added to PCPs as emollient or solvent. ${ }^{3,4}$ Some PCPs are used with a high frequency (e.g., deodorant or day cream once a day). ${ }^{5}$ Often multiple products are used simultaneously, leading to aggregate exposure (i.e., simultaneous exposure to the same substance from all possible sources and routes). ${ }^{6,7}$ For example, D4 or D5 present in a deodorant or day cream could be inhaled when applying the product and could be dermally absorbed as the product stays on the skin during the day.

The widely investigated toxicity of D4 and D5 has recently been reviewed by the Scientific Committee on Consumer Safety (SCCS) of the European Union ${ }^{4}$ and the Cosmetic Ingredient Review (CIR) expert panel in the United States. ${ }^{3}$ Both committees state that the use of D4 and D5 in PCPs represents a minimal human health risk. However, data to assess the potential health risks of aggregate exposure were not available and therefore not included in the assessment.

The majority of D4 and D5 that becomes systemically available, either through inhalation or dermal absorption, is eliminated by exhalation of the parent compound. ${ }^{8}$ A small fraction is metabolized and excreted in urine. ${ }^{9,10}$ D4 and D5 were quantified using GC-MS in different biological media, such as blood and urine. ${ }^{1-13}$ Their vapor pressures (Table 1) and low blood:air partition coefficients make them suitable for detection in end-exhaled air. ${ }^{14,15}$ Plotzke and co-workers studied ${ }^{13} \mathrm{C}-\mathrm{D} 4$ and ${ }^{13} \mathrm{C}-\mathrm{D} 5$ concentrations in exhaled air. $^{16,17}$ Tedlar bags were used for sample collection and the substances of interest were trapped on Tenax adsorbent tubes, desorbed with pure hexane, and analyzed by GC-MS. Recently, Pieri and colleagues determined the presence of linear and cyclic volatile methyl siloxanes (D3 to D6) in indoor air using TD-GC-MS. ${ }^{18}$ To our knowledge, our method is the first describing the use of TD-GC-MS as an analytical technique to quantify native cyclic siloxanes in end-exhaled air.

The concept of sampling volatile organic compounds (VOCs) in end-exhaled air is briefly introduced below. The basic concept described by Farhi ${ }^{19}$ has been adjusted as we assume that VOCs are present in ambient air, leading to

Received: February 3, 2014

Accepted: May 16, 2014

Published: May 16, 2014 
Table 1. Chemical Structure and Some Physical Chemical Properties of D4 and D5

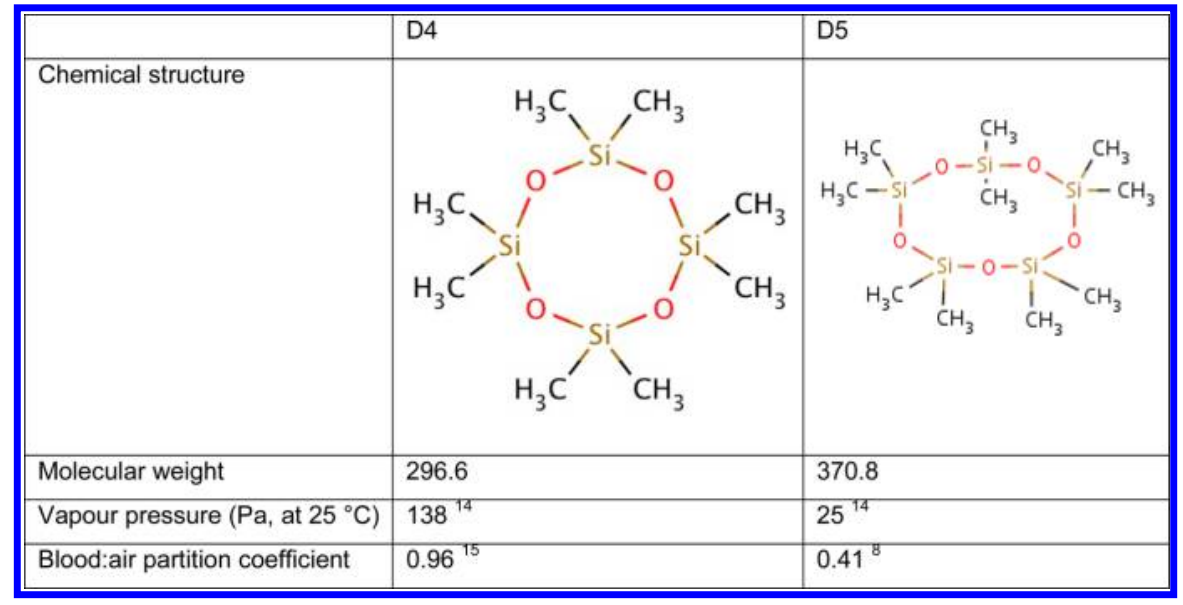

inhalation of the substance. Under steady-state conditions, the amount of VOC entering the lung via venous blood equals the difference between the amount of VOC entering and leaving the lung via inhalation or exhalation $\left(C_{\mathrm{A}}-C_{\text {in }}\right)$ and the amount of VOC leaving via arterial blood and can be described according to the following mass balance (eq 1):

$$
Q_{\mathrm{C}} \times C_{\mathrm{V}}=V_{\mathrm{A}} \times\left(C_{\mathrm{A}}-C_{\text {in }}\right)+Q_{\mathrm{C}} \times C_{\mathrm{art}}
$$

where $Q_{C}$ is the cardiac output $(\mathrm{L} / \mathrm{min}), C_{\mathrm{V}}$ is the venous blood concentration $(\mathrm{ng} / \mathrm{L}), V_{\mathrm{A}}$ is the ventilation rate $(\mathrm{L} / \mathrm{min}), C_{\mathrm{A}}$ is the alveolar concentration during exhalation $(\mathrm{ng} / \mathrm{L}), C_{\text {in }}$ the concentration in inhaled air $(\mathrm{ng} / \mathrm{L})$, and $C_{\text {art }}$ is the arterial blood concentration (ng/L).

The arterial blood concentration is depending on the blood:air partition coefficient $\left(P_{\mathrm{B}}\right)$, assuming diffusion equilibrium between arterial blood leaving the lung and alveolar air leaving the lung (eq 2):

$$
C_{\text {art }}=C_{\mathrm{A}} \times P_{\mathrm{B}}
$$

Subsequently, both eqs 1 and 2 can be combined to describe the alveolar concentration (eq 3). The alveolar concentration $\left(C_{\mathrm{A}}\right)$ is proportional to the concentration of the VOC in mixed venous blood $\left(C_{\mathrm{V}}\right)$ and the concentration of the VOC present in inhaled air $\left(C_{\text {in }}\right)$, which is associated with the substancespecific blood:air partition coefficient $\left(P_{\mathrm{B}}\right)$, alveolar ventilation $\left(V_{\mathrm{A}}\right)$, and cardiac output $\left(Q_{\mathrm{C}}\right) .{ }^{19,20}$

$$
C_{\mathrm{A}}=\frac{\left(Q_{\mathrm{C}} \times C_{\mathrm{V}}\right)+\left(V_{\mathrm{A}} \times C_{\mathrm{in}}\right)}{V_{\mathrm{A}}+Q_{\mathrm{C}} \times P_{\mathrm{B}}}
$$

When measuring VOCs with a low blood:air partition coefficient $\left(P_{\mathrm{B}} \leq 10\right)$, the concentration in venous blood is primarily influenced by the ventilation rate and cardiac output. Consequently, slight variations in these parameters will lead to inter and intra individual differences. ${ }^{21}$ In contrast, for VOCs with a high blood:air partition coefficient $\left(P_{\mathrm{B}}>10\right)$, the venous blood concentration is less affected by changes in ventilation rate and cardiac output. Water-soluble compounds may become dissolved in the mucous membrane lining the respiratory tract during inhalation and exhalation (i.e., the wash-in wash-out effect). This results in a lower uptake of the hydrophilic solvent compared to what would be expected based on blood:air partitioning. ${ }^{22,23}$ The wash-in wash-out effect requires standardization of the sample collection procedure and an adjustment for this effect should be considered when modeling the data.

The collection of samples of end-exhaled air, using canisters, bags, or glass tubes, is noninvasive, and because of easy accessibility, repeated samples can be obtained in a short time period without causing much of a burden to the study subject. ${ }^{24,25}$ This makes exhaled air analysis attractive for kinetic studies.

The aim of this study was to quantify D4 and D5 in endexhaled air, as it reflects the blood concentration and therefore internal exposure to D4 and D5. This method was used in a human exposure study focused on the dermal uptake of D4 and D5 as a pure substance and as a constituent of night cream or deodorant.

\section{MATERIALS AND METHODS}

Chemicals. Octamethylcyclotetrasiloxane (98\% D4; CAS 556-67-2) and decamethylcyclopentasiloxane (97\% D5; CAS 541-02-6) were obtained from Sigma-Aldrich (St. Louis, MO, United States). ${ }^{13} \mathrm{C}$-labeled D4 and ${ }^{13} \mathrm{C}$-labeled D5, used as internal standards, were purchased from Dow Corning (Midland, MI, United States). Methanol (99.9\%; CAS 67-56-1) supplied by Boom (Meppel, The Netherlands) was used to dissolve ${ }^{13} \mathrm{C}$-labeled D4 or D5 and unlabeled D4 or D5 for the preparation of standards. Bio-VOC breath samplers were obtained from Markes International (Llantrisant, United Kingdom) and automatic thermal desorption (ATD) 0.25 in. $\times 3.5$ in. stainless steel tubes filled with Carbograph 2 TD 60/80 and Carbograph 1 TD 60/80 were obtained from Camsco (Houston, TX).

Standard Preparation. We prepared a stock solution of $10 \mu \mathrm{g} / \mu \mathrm{L}$ of D4 and D5 in methanol. From this solution standards were prepared of $10,5,2,1,0.5$, and $0.1 \mathrm{ng} / \mu \mathrm{L} .{ }^{13} \mathrm{C}$-labeled $\mathrm{D} 4$ and ${ }^{13} \mathrm{C}$-labeled D5 were diluted with $10 \mathrm{~mL}$ of methanol to a stock of $1 \mu \mathrm{g} / \mu \mathrm{L}$. Both stock solutions were further diluted and combined to a working solution of $5 \mathrm{ng} / \mu \mathrm{L}$.

Sample Collection and Preparation. Prior to use, the ATD tubes were conditioned at least $4 \mathrm{~h}$ at $350{ }^{\circ} \mathrm{C}$ using a multitube conditioner (TC-20, Markes International, Llantrisant, United Kingdom). End-exhaled breath samples were collected using Bio-VOC breath samplers. The participants were instructed to inhale normally, to place the Bio-VOC breath sampler into their mouth and to exhale fully at normal speed into the sampler. Immediately following sample collection, the substances of interest were transferred to an ATD tube using the syringe 


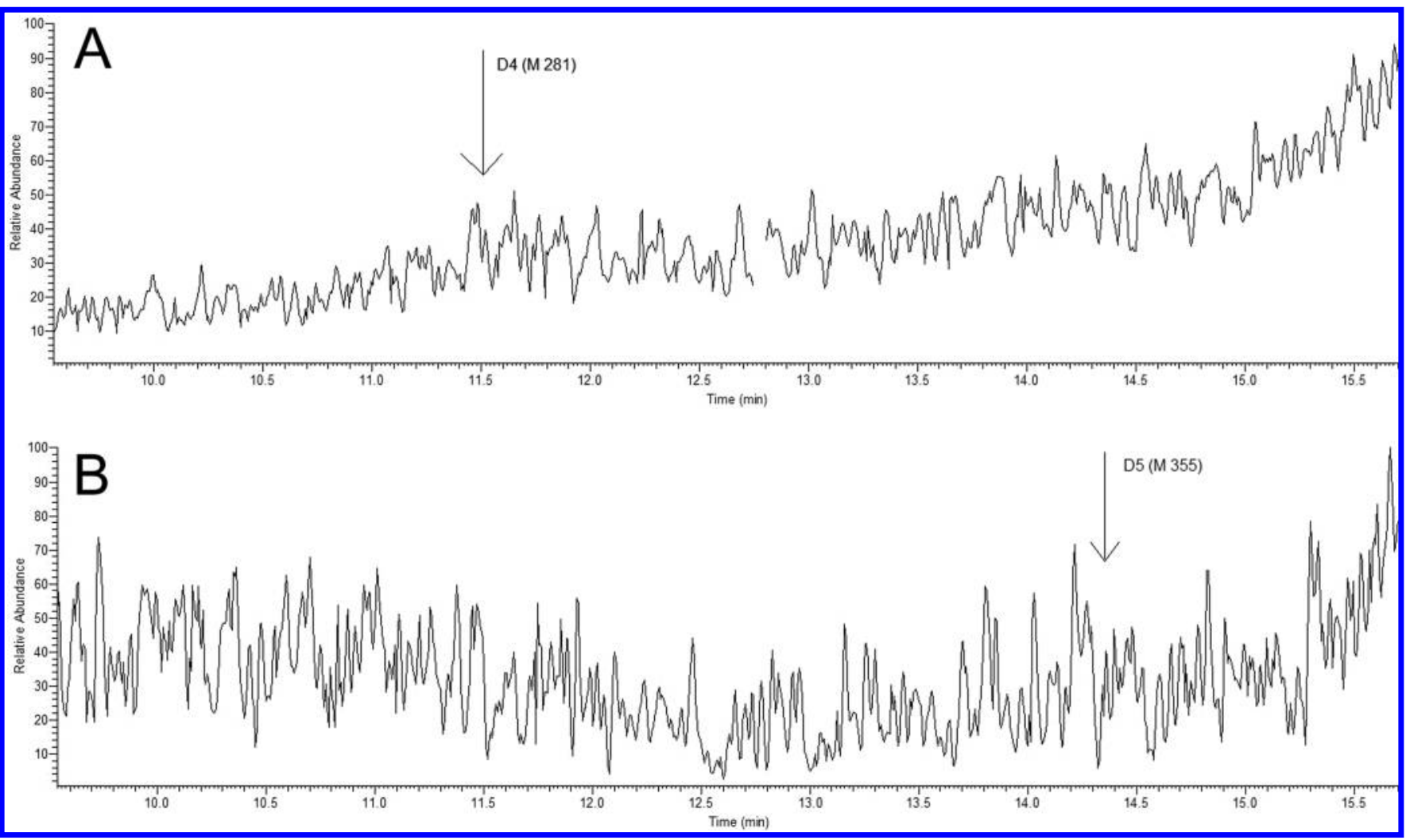

Figure 1. Extracted ion chromatogram of D4 (A) and D5 (B) after analysis of the TD-GC-MS system without the presence of samples loaded with D4 and D5.

(plunger). The ATD tubes were capped with Swagelock-caps and stored until analysis at ambient temperature for less than 24 h. Subsequently, $2.5 \mathrm{ng}$ of ${ }^{13} \mathrm{C}$-labeled D4 and D5 in $0.5 \mu \mathrm{L}$ of methanol was loaded on the ATD tubes using a loading rig (Markes International, Llantrisant, United Kingdom). The ATD tube was connected to the loading rig, the internal standard solution was injected using a syringe, and the tube was flushed with helium 5.0 (Linde Gas, Schiedam, The Netherlands) at a flow of $50 \mathrm{~mL} / \mathrm{min}$ for $3 \mathrm{~min}$ to remove the methanol.

Calibration Procedure and Quantification. Calibration and quantification was carried out by spiking seven ATD tubes in a concentration range between 0 and $10 \mathrm{ng} / \mu \mathrm{L}$ D4 and D5. A conditioned ATD tube was connected to the loading rig. Next, the standards were loaded on the tubes using a syringe and flushing the tube with helium 5.0 at a flow of $50 \mathrm{~mL} / \mathrm{min}$ for $3 \mathrm{~min}$ to remove the methanol. Subsequently, this procedure was repeated for loading of the internal standards. Linear calibration curves were constructed by plotting the quotient of the peak area of the analytes of interest and the corresponding labeled internal standards versus the standard concentrations. The calibration curves routinely had $r^{2} \geq 0.99$ for both substances.

Analysis. The samples were analyzed by use of thermal desorption gas chromatography mass spectrometry (TD-GC-MS). The instrument consisted of a thermal desorption unit and auto sampler (Unity 2 and Ultra 2, Markes) coupled to a gas chromatograph mass spectrometer (Focus and ISQ Thermo Scientific, Interscience Breda, The Netherlands). Electron impact ionization was used. The ATD tubes were inserted in the auto sampler and subsequently desorbed at $275^{\circ} \mathrm{C}$ for $15 \mathrm{~min}$, using a split flow of $10 \mathrm{~mL} / \mathrm{min}$. Samples were trapped at $-10{ }^{\circ} \mathrm{C}$ by using a general purpose hydrophobic trap (U-T2GPH-2S). A $30 \mathrm{~m}$ Rxi-5 MS (0.25 mm i.d., $0.5 \mu \mathrm{m}$ film thickness, Restek) was used as an analytical column. Helium 5.0 was used as carrier gas. The GC oven temperature was programmed as follows: hold for 5 min at $50{ }^{\circ} \mathrm{C} ; 10^{\circ} \mathrm{C} / \mathrm{min}$ to $150{ }^{\circ} \mathrm{C} ; 30^{\circ} \mathrm{C} / \mathrm{min}$ to $250{ }^{\circ} \mathrm{C}$, hold for $2 \mathrm{~min}$. The transfer line was kept at $250^{\circ} \mathrm{C}$ and the ion source at $250{ }^{\circ} \mathrm{C}$. We ran in full scan mode and the ions monitored were $\mathrm{m} / z 281$ for D4, 355 for D5, 285 for ${ }^{13} \mathrm{C}$-labeled D4, and 360 for ${ }^{13}$ C-labeled D5, respectively. The dwell time was set at $0.4 \mathrm{~s}$.

Biological Monitoring. To demonstrate the applicability of the method for quantifying values at a low range, we collected end-exhaled air samples $(142 \mathrm{~mL})$ in duplicate from 15 consumers exposed to PCPs (regular use) and from the same consumers after they refrained from the use of PCPs for $24 \mathrm{~h}$. Regular use was described as the use of PCPs by our volunteers as they would normally do without restrictions.

\section{RESULTS AND DISCUSSION}

Prevention of Contamination. The ubiquitous presence of cyclic siloxanes and their unique chemistry makes their analysis at trace levels challenging. ${ }^{26}$ Several measures were taken to prevent contamination of the analytical process: (1) D4 and D5 are present in a wide variety of PCPs, such as hand creams and deodorants. All researchers, lab technicians, and instrument operators refrained from the use of these products prior to $(24 \mathrm{~h})$ and during the experimental and analytical work. (2) All calibration and internal standard solutions were prepared in glass jars with metal-lined screw caps to prevent contamination of the solutions due to contact with plastic (possible silicon-containing) screw caps. (3) The initial pressure regulator, regulating helium flow, was replaced by a pressure regulator with a complete iron inner lining. (4) All parts of the TD-GC-MS system that contained silicon were replaced by silicon-free alternatives (e.g., septa). Before the start of every 
analysis the system was checked with an empty tube, which did not contain a sorbent, to verify that the system was not a source of background contamination (Figure 1A,B).

Conditioning. ATD tubes, conditioned by the supplier with nitrogen, appeared to contain substantial amounts of D4 and D5 upon arrival. Helium 4.5 was used to condition the ATD tubes, but the D4 and D5 levels present on the tubes did not decrease. To improve the precleaning efficiency, helium 4.5 was replaced by helium of superior quality (helium 5.0). Despite extensive conditioning, we were unable to completely remove all D4 and D5. Conditioning was considered satisfactory if the residual background level for both substances was present on the tubes, corresponding to $<69 \mathrm{pg} /$ tube for D4 and $<47 \mathrm{pg} /$ tube for D5. As the analytical system itself was not a source of contamination, we suggest that D4 and D5 were difficult to remove completely as they originated from the adsorbent material in the tubes. We accounted for this problem by analyzing each individual conditioned tube and subtracting the peak areas of the analytes on the tube prior to sample collection from the peak areas observed in the final analysis of a tube loaded with end-exhaled air.

Quantification limit. The limit of quantification (LOQ) was determined to be the concentration at which the repeatability has a coefficient of variation of $\leq 25 \%$ (Figure 2 ).

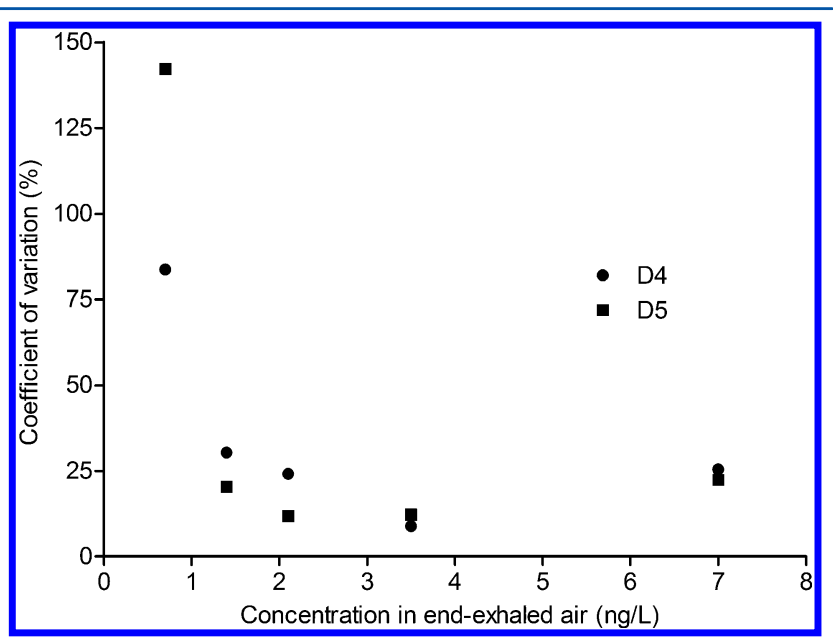

Figure 2. Coefficient of variation versus the D4 and D5 concentration in end-exhaled air.

The LOQ was $2.1 \mathrm{ng} / \mathrm{L}$ end-exhaled air (0.3 ng/tube) for D4 and $1.4 \mathrm{ng} / \mathrm{L}$ end-exhaled air ( $0.2 \mathrm{ng} /$ tube $)$ for D5.

Repeatability of the Method. This method shows well resolved peaks without interference (Figure 3A,B).

Repeatability for both substances improved due to the addition of an internal standard. The coefficient of variation decreased from $30.8 \%$ to $9.5 \%$ for D4 and from $37.8 \%$ to $12.5 \%$ for D5. Over a period of months, the slope of the calibration curves showed a small variation as the coefficient of variation was $7.0 \%$ for D4 and $10.4 \%$ for D5. To take this variation in account, in each series of samples standards were analyzed. The inter- and intraday variations were similar for both substances (Table 2).

Breakthrough and Collection Efficiency. With this experimental setup, it was unlikely that breakthrough of D4 and D5 would occur as the concentrations present in the endexhaled air samples were rather low. The siloxanes collected using the Bio-VOC breath sampler were transferred to the ATD tubes with a mean efficiency of $96 \%( \pm 3 \%)$.
Table 2. Inter- and Intraday Variability Expressed as Coefficient of Variation

\begin{tabular}{ccccccccc} 
& \multicolumn{3}{c}{ interday } & & \multicolumn{3}{c}{ intraday } \\
\cline { 2 - 4 } \cline { 7 - 8 } concentration (ng/tube) & $N$ & D4 (\%) & D5 (\%) & & D4 (\%) & D5(\%) \\
1 & 17 & 22.7 & 15.9 & & 5 & 25.4 & 22.4 \\
5 & 16 & 8.8 & 12.8 & & 6 & 9.5 & 12.5
\end{tabular}

Table 3. Mean Concentrations of D4 and D5 in End-Exhaled Air Collected from Human Volunteers Who Used PCPs and from the Same Study Participants Not Using PCPs for $24 \mathrm{~h}$ Prior to Sample Collection

\begin{tabular}{|c|c|c|c|c|}
\hline \multirow[b]{2}{*}{ D4 (ng/L) } & \multicolumn{2}{|c|}{ regular use of PCPs } & \multicolumn{2}{|c|}{$\begin{array}{l}\text { no use of PCPs for } 24 \mathrm{~h} \text { prior } \\
\text { to sample collection }\end{array}$} \\
\hline & first sample & second sample & first sample & second sample \\
\hline 1 & 2.1 & 4.3 & 2.8 & 3.8 \\
\hline 2 & 7.7 & 6.4 & $<2.1$ & $<2.1$ \\
\hline 3 & 9.1 & 20.0 & $<2.1$ & 2.9 \\
\hline 4 & $2.7^{a}$ & 10.8 & $<2.1$ & 4.0 \\
\hline 5 & 2.4 & 3.9 & 2.5 & $<2.1$ \\
\hline 6 & 2.1 & 3.3 & $<2.1$ & $<2.1$ \\
\hline 7 & 3.4 & 5.2 & $<2.1$ & $<2.1$ \\
\hline 8 & 2.4 & $<2.1$ & $8.7^{a}$ & $<2.1$ \\
\hline 9 & $3.8^{a}$ & 13.1 & $<2.1$ & $<2.1$ \\
\hline 10 & $<2.1$ & 2.5 & 3.1 & $<2.1$ \\
\hline 11 & 18.0 & 5.6 & $<2.1$ & $<2.1$ \\
\hline 12 & $11.2^{a}$ & 43.5 & $<2.1$ & $<2.1$ \\
\hline 13 & 10.9 & 2.2 & $<2.1$ & $<2.1$ \\
\hline 14 & 2.3 & 3.4 & 2.2 & $<2.1$ \\
\hline 15 & 86.4 & $3.1^{a}$ & $<2.1$ & $<2.1$ \\
\hline \multirow[b]{2}{*}{ D5 (ng/L) } & \multicolumn{2}{|c|}{ regular use of PCPs } & \multicolumn{2}{|c|}{$\begin{array}{l}\text { no use of PCPs for } 24 \mathrm{~h} \text { prior } \\
\text { to sample collection }\end{array}$} \\
\hline & first sample & second sample & first sample & second sample \\
\hline 1 & 2.9 & 3.4 & 5.2 & 1.8 \\
\hline 2 & 9.2 & 3.9 & $<1.4$ & $<1.4$ \\
\hline 3 & 23.2 & 13.3 & 3.3 & $<1.4$ \\
\hline 4 & 1.4 & 1.8 & 5.8 & 2.0 \\
\hline 5 & 2.0 & 1.7 & $<1.4$ & 3.3 \\
\hline 6 & 2.7 & 3.0 & $<1.4$ & $<1.4$ \\
\hline 7 & $17.0^{a}$ & 37.3 & 1.9 & 2.8 \\
\hline 8 & 3.7 & 1.9 & $<1.4^{a}$ & 18.5 \\
\hline 9 & $4.9^{a}$ & 83.8 & $<1.4$ & $<1.4$ \\
\hline 10 & $<1.4$ & 4.7 & $<1.4$ & 1.8 \\
\hline 11 & 3.2 & $<1.4$ & $<1.4$ & $<1.4$ \\
\hline 12 & 10.4 & 55.9 & $<1.4$ & $<1.4$ \\
\hline 13 & 3.0 & $<1.4$ & $<1.4$ & 1.7 \\
\hline 14 & 6.8 & 2.1 & $<1.4$ & $<1.4$ \\
\hline 15 & 34.1 & $1.6^{a}$ & $<1.4$ & $<1.4$ \\
\hline
\end{tabular}

${ }^{a}$ Note that there is a rather large difference between the two samples collected. The lowest value is probably not representing the D4 or D5 concentration in alveolar air, as the volunteer did not exhale completely. The value is representing the D4 or D5 concentration present in air which consists of a combination of air present in the death space of the lungs and in the alveoli.

Presence of Water in End-Exhaled Air Samples. In a recent article by Guallar-Hoyas and co-workers, ${ }^{27}$ it was suggested that the presence of water in breath samples, when thermally desorbed, could damage the stationary phase of the GC column and lead to the production of cyclic siloxanes due to hydrolysis. Consequently, the retention times and separations were not reproducible across analytical campaigns. 


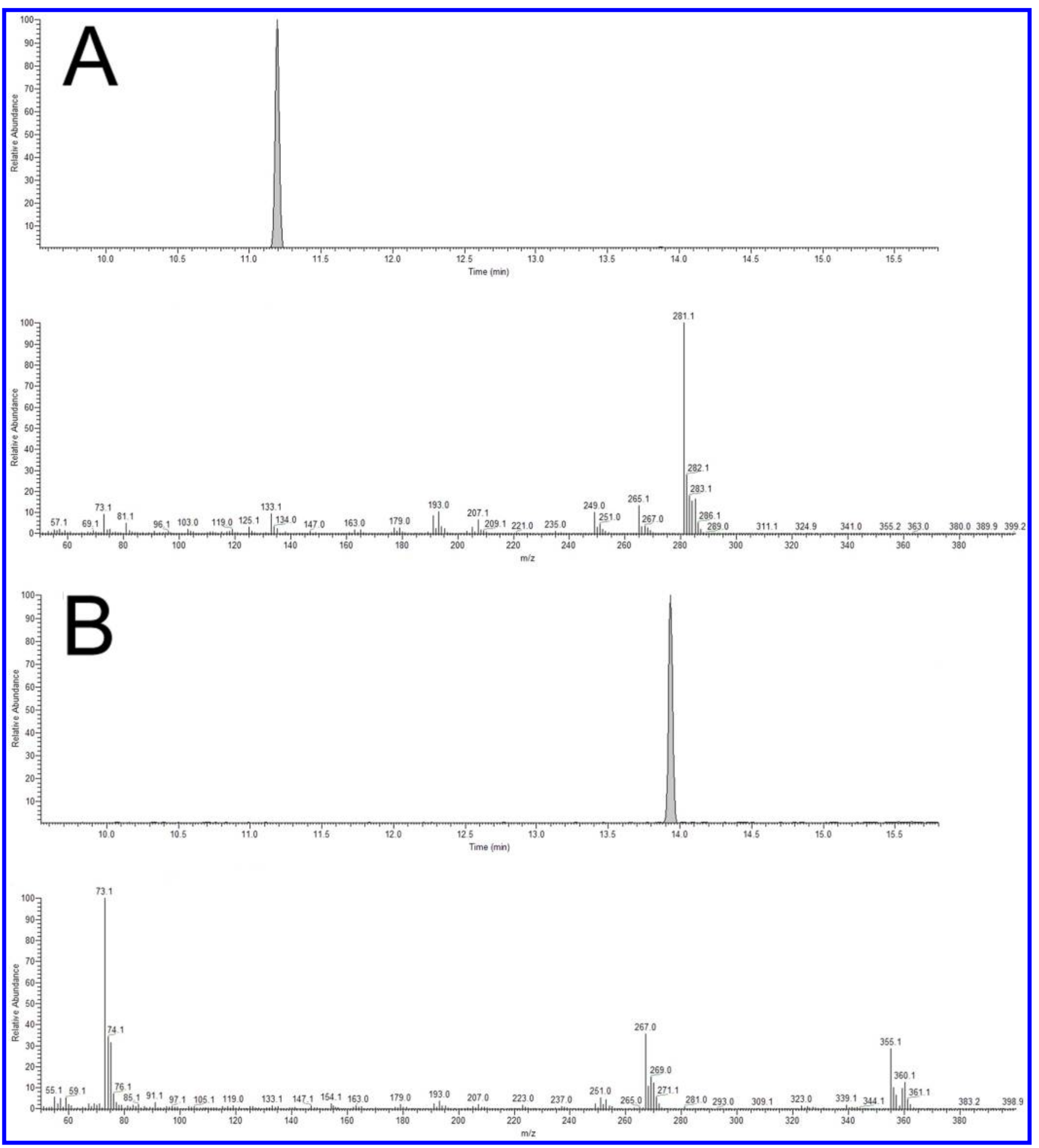

Figure 3. TD-GC-MS chromatogram and the EI-mass spectra of D4 (A) and D5 (B), analysis of a sample provided by a volunteer who was exposed to PCPs on a regular basis.

We believe that the presence of water in our end-exhaled air samples did not interfere with the stationary phase. The method presented in this paper has been used to assess the D4 and D5 content of approximately 1300 end-exhaled air samples obtained in various experiments. The samples have been analyzed between March and August 2013 and the retention times were stable during this period. Furthermore, when loading the internal standard, the tube was purged with helium for $3 \mathrm{~min}$, and directly at the start of the TD-GC-MS program, the tube was dry-purged for $1 \mathrm{~min}$ to remove excess water.

Results of Biological Monitoring. Table 3 shows the results of end-exhaled air samples, collected from 15 volunteers who were using PCPs (regular use) and from the same consumers who refrained from the use of PCPs for $24 \mathrm{~h}$. The cyclic siloxanes investigated were indicated on the ingredient labels of the PCPs used by the volunteers. All samples were 
collected in duplicate. The mean concentration in end-exhaled air ranged from 2 to $45 \mathrm{ng} / \mathrm{L}$ for D4 and from 2 to $44 \mathrm{ng} / \mathrm{L}$ for D5 following regular use of PCPs. The mean concentration of the cyclic siloxanes in end-exhaled air of consumers who did not apply any PCPs for $24 \mathrm{~h}$ was below $4 \mathrm{ng} / \mathrm{L}$ for D4 and below $9 \mathrm{ng} / \mathrm{L}$ for D5. When volunteers refrained from the use of PCPs $24 \mathrm{~h}$ prior to sample collection, the mean D4 and D5 concentration in end-exhaled air was, in most cases, similar or lower compared to the mean concentration measured after regular use of PCPs. Table 3 shows one exception (volunteer number 8 ), which may be explained by inhalation exposure to ambient sources of D4 and D5 while using public transportation to our laboratory. A significant difference was found between the D4 concentration in end-exhaled air of volunteers who did not use personal care products for $24 \mathrm{~h}$ and of the same volunteers who regularly used PCPs $(P=0.009)$. For D5 also a difference was found, but failed to reach the $5 \%$ level of significance $(P=0.078)$, probably because of the small sample size.

\section{CONCLUSION}

Analysis of end-exhaled air represents a new and promising noninvasive approach in the use of D4 and D5 as chemical markers of cyclic siloxanes in consumer and environmental exposure assessment. Cyclic siloxanes (D4 and D5) can be quantified in end-exhaled air at concentration levels as low as background levels observed in the general population. To our knowledge, this is the first method describing the use of TD-GC-MS as an analytical technique to quantify cyclic siloxanes in end-exhaled air.

\section{AUTHOR INFORMATION}

\section{Corresponding Author}

*E-mail: Jacqueline.Biesterbos@radboudumc.nl. Telephone: +31-24-3613728. Fax: +31-24-3613505.

Notes

The authors declare no competing financial interest.

\section{REFERENCES}

(1) Horii, Y.; Kannan, K. Arch. Environ. Contam. Toxicol. 2008, 55, 701-710.

(2) Wang, R.; Moody, R. P.; Koniecki, D.; Zhu, J. Environ. Int. 2009, $35,900-904$.

(3) Johnson, W., Jr.; Bergfeld, W. F.; Belsito, D. V.; Hill, R. A.; Klaassen, C. D.; Liebler, D. C.; Marks, J. G., Jr.; Shank, R. C.; Slaga, T. J.; Snyder, P. W.; Andersen, F. A. Int. I. Toxicol. 2011, 30, 149S-227S.

(4) Scientific Committee on Consumer Safety (SCCS), Opinion on cyclomethicone (D4/D5); 2010, http://ec.europa.eu/health/scientific committees/consumer safety/docs/sccs_o 029.pdf, accessed on 1-2-2013.

(5) Biesterbos, J. W.; Dudzina, T.; Delmaar, C. J.; Bakker, M. I.; Russel, F. G.; von Goetz, N.; Scheepers, P. T.; Roeleveld, N. Food Chem. Toxicol. 2013, 55, 8-17.

(6) Lorenz, C.; Von Goetz, N.; Scheringer, M.; Wormuth, M.; Hungerbuhler, K. Nanotoxicology 2011, 5, 12-29.

(7) von Goetz, N.; Wormuth, M.; Scheringer, M.; Hungerbuhler, K. Risk Anal 2010, 30, 473-487.

(8) Reddy, M. B.; Dobrev, I. D.; McNett, D. A.; Tobin, J. M.; Utell, M. J.; Morrow, P. E.; Domoradzki, J. Y.; Plotzke, K. P.; Andersen, M. E. Toxicol. Sci. 2008, 105, 275-285.

(9) Varaprath, S.; McMahon, J. M.; Plotzke, K. P. Drug Metab. Dispos. 2003, 31, 206-214.

(10) Varaprath, S.; Salyers, K. L.; Plotzke, K. P.; Nanavati, S. Drug Metab. Dispos. 1999, 27, 1267-1273.

(11) Flassbeck, D.; Pfleiderer, B.; Grumping, R.; Hirner, A. V. Anal. Chem. 2001, 73, 606-611.
(12) Hanssen, L.; Warner, N. A.; Braathen, T.; Odland, J. O.; Lund, E.; Nieboer, E.; Sandanger, T. M. Environ. Int. 2013, 51, 82-87.

(13) Varaprath, S.; Seaton, M.; McNett, D. A.; Cao, L.; Plotzke, K. P. Int. I. Environ. Anal. Chem. 2000, 77, 16.

(14) Flaningam, O. L. I. Chem. Eng. Data 1986, 31, 266-272.

(15) Reddy, M. B.; Andersen, M. E.; Morrow, P. E.; Dobrev, I. D.; Varaprath, S.; Plotzke, K. P.; Utell, M. I. Toxicol. Sci. 2003, 72, 3-18.

(16) Plotzke, K. P.; Utell, M. J.; Looney, R. J. Absorption, Distribution and Elimination of ${ }^{13} \mathrm{C}-\mathrm{D} 4$ in Humans After Dermal Administration, EPA document 86010000007, 2000.

(17) Plotzke, K. P.; Utell, M. J.; Looney, R. J. Absorption, Distribution and Elimination of 13C-D5 in Humans After Dermal Administration, EPA document 84030000008, 2002.

(18) Pieri, F.; Katsoyiannis, A.; Martellini, T.; Hughes, D.; Jones, K. C.; Cincinelli, A. Environ. Int. 2013, 59, 363-371.

(19) Farhi, L. E. Respir. Phvsiol. 1967, 3, 1-11.

(20) Fiserova-Bergerova, V. In Modeling of inhalation exposure to vapors: uptake, distribution and elimination; Fiserova-Bergerova, V., Ed.; CRC Press: Boca Raton, FL, 1983.

(21) King, J.; Unterkofler, K.; Teschl, G.; Teschl, S.; Koc, H.; Hinterhuber, H.; Amann, A. I. Math. Biol. 2011, 63, 959-999.

(22) Johanson, G. Ann. Occup. Hvg. 1991, 35, 323-339.

(23) Mork, A. K.; Johanson, G. Toxicol. Lett. 2006, 164, 6-15.

(24) Alonso, M.; Sanchez, J. M. TrAC, Trends Anal. Chem. 2013, 44, $78-89$.

(25) Kim, K. H.; Jahan, S. A.; Kabir, E. TrAC, Trends Anal. Chem. 2012, 33, 1-8.

(26) Varaprath, S.; Stutts, D.; Kozerski, G. Silicon Chem. 2006, 3, 23.

(27) Guallar-Hoyas, C.; Turner, M. A.; Blackburn, G. J.; Wilson, I. D.; Thomas, C. L. P. Bioanalvsis 2012, 4, 2227-2237. 